\title{
Point source confusion in SZ cluster surveys
}

\author{
J. G. Bartlett ${ }^{1}$ and J.-B. Melin ${ }^{2}$
}

1 APC, 11 pl. Marcelin Berthelot, 75231 Paris Cedex 05, France

(UMR 7164 CNRS, Université Paris 7, CEA, Observatoire de Paris)

e-mail: bartlett@cdf.in2p3.fr

2 Department of Physics, University of California Davis, One Shields Avenue, Davis, CA, 95616 USA

e-mail: melin@bubba.physics.ucdavis.edu

Received 10 May 2005 / Accepted 20 September 2005

\begin{abstract}
We examine the effect of point source confusion on cluster detection in Sunyaev-Zel'dovich (SZ) surveys. A filter matched to the spatial and spectral characteristics of the SZ signal optimally extracts clusters from the astrophysical backgrounds. We calculate the expected confusion (point source and primary cosmic microwave background $[\mathrm{CMB}]$ ) noise through this filter and quantify its effect on the detection threshold for both single and multiple frequency surveys. Extrapolating current radio counts, we estimate that confusion from sources below $\sim 100 \mu \mathrm{Jy}$ limits single-frequency surveys to $1 \sigma$ detection thresholds of $Y \sim 3 \times 10^{-6} \operatorname{arcmin}^{2}$ at $30 \mathrm{GHz}$ and $Y \sim 10^{-5} \operatorname{arcmin}^{2}$ at $15 \mathrm{GHz}$ (for unresolved clusters in a 2 arcmin beam); these numbers are highly uncertain, and an extrapolation with flatter counts leads to much lower confusion limits. Bolometer surveys must contend with an important population of infrared point sources. We find that a three-band matched filter with 1 arcmin resolution (in each band) efficiently reduces confusion, but does not eliminate it: residual point source and CMB fluctuations contribute significantly to the total filter noise. In this light, we find that a 3-band filter with a low-frequency channel (e.g, $90+150+220 \mathrm{GHz})$ extracts clusters more effectively than one with a high frequency channel (e.g, $150+220+300 \mathrm{GHz}$ ).
\end{abstract}

Key words. cosmic microwave background - galaxies: clusters: general - methods: observational

\section{Introduction}

Galaxy cluster surveys based on the Sunyaev-Zel'dovich (SZ) effect (Sunyaev \& Zeldovich 1970, 1972; for comprehensive reviews, see Birkinshaw 1999 and Carlstrom et al. 2002) will soon supply large, homogeneous catalogs out to redshifts well beyond unity (Barbosa et al. 1996; Eke et al. 1996; Colafrancesco et al. 1997; Holder et al. 2000; Bartlett 2001; Kneissl et al. 2001; Benson et al. 2002). Eagerly awaited, these surveys will probe dark energy and its evolution, and shed new light on galaxy formation (Haiman et al. 2001; Weller \& Battye 2003; Wang et al. 2004). The instruments designed for these observations are of two types: dedicated interferometer arrays surveying at a single frequency (e.g., AMI, AMiBA, and SZA) and bolometer systems operating over several millimeter bands (e.g., ACBAR, ACT, APEX, Olimpo, Planck, SPT $)^{1}$. Groundbased and balloon-borne instruments are expected to find up to several thousands of clusters, while the Planck mission will produce an essentially all-sky catalog of several $10^{4}$ clusters by the end of the decade.

${ }^{1}$ A list of experiment web pages is given at the end of the reference section.
An important issue facing these surveys is cluster detection in the presence of other astrophysical foregrounds/backgrounds. Except for the very nearby ones, clusters will appear as extended sources over arcminute scales. Power in diffuse Galactic emission (synchrotron, free-free and dust emission) and in the primary cosmic microwave background (CMB) anisotropy falls on these scales and the clusters can be efficiently extracted using an adapted spatial filter (Haehnelt \& Tegmark 1996; Herranz et al. 2002; Schäfer et al. 2004); fluctuations caused by point sources (radio and infrared galaxies), on the other hand, are important on these scales and represent a potentially serious source of confusion for SZ cluster searches (Knox et al. 2004; White \& Majumdar 2004; Aghanim et al. 2005).

The two kinds of SZ survey instruments deal with this problem in different ways. Single frequency surveys must individually identify and remove point sources with high angular resolution (better than 1 arcmin) observations, which interferometers achieve by incorporating several long baselines in their antenna array. Operating at relatively low frequencies $(15 \mathrm{GHz}$ for AMI, $30 \mathrm{GHz}$ for the SZA and $90 \mathrm{GHz}$ for AMiBA), these surveys will contend with the radio galaxy population. Bolometer-based instruments will not have the angular 
resolution needed to spatially separate point sources from galaxy clusters; they must instead rely on spectral information. In their millimeter wavelength bands, these instruments will contend with the poorly known far-infrared point source population.

In this paper we quantify the effect of point source confusion on cluster detection in SZ surveys. We shall only consider the effect of the random field population, but we note that point sources are expected to preferentially reside in the clusters themselves, locally raising the effective noise level; we leave examination of this effect to a future work. White \& Majumdar (2004) recently calculated the expected confusion due to IR point sources as a function of frequency, while Knox et al. (2004) and Aghanim et al. (2005) studied their influence on the measurement of SZ cluster parameters. We extend this work by considering cluster detection with an optimal filter spatially and spectrally (for multi-frequency surveys) matched to the thermal SZ signal (Haehnelt \& Tegmark 1996; Herranz et al. 2002; Schäfer et al. 2004). Using the matched filter, we quantify the confusion noise induced by extragalactic point sources for both single-frequency radio and multifrequency bolometer SZ surveys.

We begin by briefly describing our matched filter and cluster detection routine, leaving details to Melin et al. (2005). In Sect. 3 we present our point source model, based on recent number counts in the radio and far-infrared. We then calculate the confusion noise through the filter to examine its importance for future SZ surveys (Sect. 4). In the last section, we summarize our main results and discuss implications for SZ surveying.

\section{Detecting clusters: matched SZ filters}

The SZ effect is caused by the hot gas $(T \sim 1-10 \mathrm{keV})$ contained in galaxy clusters known as the intracluster medium (ICM); electrons in this gas up-scatter CMB photons and create a unique spectral distortion that is negative at radio wavelengths and positive in the submillimeter, with a zero-crossing near $220 \mathrm{GHz}$. The form of this distortion is universal (in the non-relativistic limit applicable to most clusters), while the amplitude is given by the Compton $y$ parameter, an integral of the gas pressure along the line-of-sight. In a SZ survey, clusters will appear as sources extended over arcminute scales (apart from the very nearby objects, which are already known) with brightness profile

$\Delta i_{v}(\boldsymbol{x})=y(\boldsymbol{x}) j_{v}$

relative to the mean CMB brightness. Here $y(\boldsymbol{x})$ is the Compton $y$ parameter at position $\boldsymbol{x}$ (a $2 \mathrm{D}$ vector on the sky) and $j_{v}$ is the SZ spectral function evaluated at the observation frequency $v$.

A SZ survey will produce maps of the sky at one or more frequencies ${ }^{2}$. We model the survey data as a vector field $\boldsymbol{M}(\boldsymbol{x})$

\footnotetext{
${ }^{2}$ Interferometers actually observe visibilities in the Fourier plane, although in this paper we will model interferometer data by its imageplane map. This should be a reasonable approximation if sampling in the Fourier plane is sufficiently good. However, it should be emphasized that the question of which space is best suited for cluster detection is important and currently under study.
}

whose components correspond to these maps (for a singlefrequency survey, the data is a scalar field). It is a sum of cluster profiles at positions $\boldsymbol{x}_{i}$ plus noise and foregrounds: $\boldsymbol{M}(\boldsymbol{x})=\sum_{i} y_{i}\left(\boldsymbol{x}-\boldsymbol{x}_{i}\right) \boldsymbol{j}_{\boldsymbol{v}}+\boldsymbol{N}(\boldsymbol{x})$, where $\boldsymbol{j}_{\boldsymbol{v}}$ is the column vector with components given by $j_{v}$ evaluated at the different observation frequencies. The vector $N(x)$ includes all non-SZ signals as well as instrumental noise; we model it as a stationary random variable with zero mean: $\langle N\rangle_{N}=0$, where the average is taken over realizations of both the instrumental noise and foreground fields. We thus assume that the mean intensity of the map is zero, i.e., that the zero mode has been taken out by the observations ${ }^{3}$. Although the model of a stationary random variable applies to the primary CMB anisotropy and (perhaps) the noise, one may question its suitability for Galactic foregrounds; it does, all the same, seem a reasonable approximation for fluctuations around the mean foreground intensity over angular scales pertinent to galaxy cluster detection (arcminutes).

We now wish to use both spatial and spectral information (when available) to best extract clusters from our survey. Consider a cluster of characteristic size $\theta$ (in the following, we take this to be the core radius of a $\beta$-profile) and central $y$-value $y_{\mathrm{o}}$ situated at an arbitrary point $\boldsymbol{x}_{i}$ on the sky. We build a filter $\boldsymbol{\Psi}_{\boldsymbol{\theta}}(\boldsymbol{x})$ (in general a column vector in frequency space) that returns an estimate, $\hat{y}_{\mathrm{o}}$, of $y_{\mathrm{o}}$ when centered on the cluster:

$\hat{y}_{\mathrm{o}}=\int \mathrm{d}^{2} x \boldsymbol{\Psi}_{\boldsymbol{\theta}}{ }^{t}\left(\boldsymbol{x}-\boldsymbol{x}_{i}\right) \cdot \boldsymbol{M}(\boldsymbol{x})$

where superscript $t$ indicates a transpose (with complex conjugation when necessary). Suppose that clusters of this characteristic size are described by an average angular form $T_{\theta}(\boldsymbol{x})$, by which we mean: $T_{\theta}(\boldsymbol{x})=\left\langle y(\boldsymbol{x}) / y_{\mathrm{o}}\right\rangle_{C}$, where the average is over many clusters of size $\theta$. We match the filter to this angular form $T_{\theta}$ and to the spectral shape of the SZ signal, requiring an unbiased estimate of the central $y$ value: $\left\langle\hat{y}_{\mathrm{o}}\right\rangle=y_{\mathrm{o}}$, where now the average is over both total noise and cluster (of size $\theta$ ) ensembles. The filter is then uniquely determined by further demanding a minimum variance estimate.

The result expressed in Fourier space (the flat sky approximation is reasonable on cluster angular scales) is (Haehnelt \& Tegmark 1996; Herranz et al. 2002; Melin et al. 2005, 2006):

$\boldsymbol{\Psi}_{\boldsymbol{\theta}}(\boldsymbol{k})=\sigma_{\theta}^{2} \boldsymbol{P}^{-1}(\boldsymbol{k}) \cdot \boldsymbol{F}_{\boldsymbol{\theta}}(\boldsymbol{k})$

where

$\boldsymbol{F}_{\theta}(\boldsymbol{k}) \equiv \boldsymbol{j}_{v} T_{\theta}(\boldsymbol{k})$

$\sigma_{\theta} \equiv\left[\int \mathrm{d}^{2} k \boldsymbol{F}_{\boldsymbol{\theta}}^{t}(\boldsymbol{k}) \cdot \boldsymbol{P}^{-1} \cdot \boldsymbol{F}_{\boldsymbol{\theta}}(\boldsymbol{k})\right]^{-1 / 2}$

with $\boldsymbol{P}(\boldsymbol{k})$ being the noise power spectrum, a matrix in frequency space with components $P_{i j}$ defined by $\left\langle N_{i}(\boldsymbol{k}) N_{j}^{*}\left(\boldsymbol{k}^{\prime}\right)\right\rangle_{N}=P_{i j}(\boldsymbol{k}) \delta\left(\boldsymbol{k}-\boldsymbol{k}^{\prime}\right)$. Note that this last expression treats, again, the astrophysical foregrounds as stationary fields. The quantity $\sigma_{\theta}^{2}$ gives the the total noise variance through the filter. We write the noise power spectrum as a sum $P_{i j}=P_{i}^{\text {noise }} \delta_{i j}+B_{i}(\boldsymbol{k}) B_{j}^{*}(\boldsymbol{k}) P_{i j}^{\text {sky }}$, where $P_{i}^{\text {noise }}$ represents the instrumental noise power, $B(\boldsymbol{k})$ the observational

\footnotetext{
${ }^{3}$ In principle this affects the measured cluster $Y$ values, but in practice any effect is small for upcoming surveys.
} 

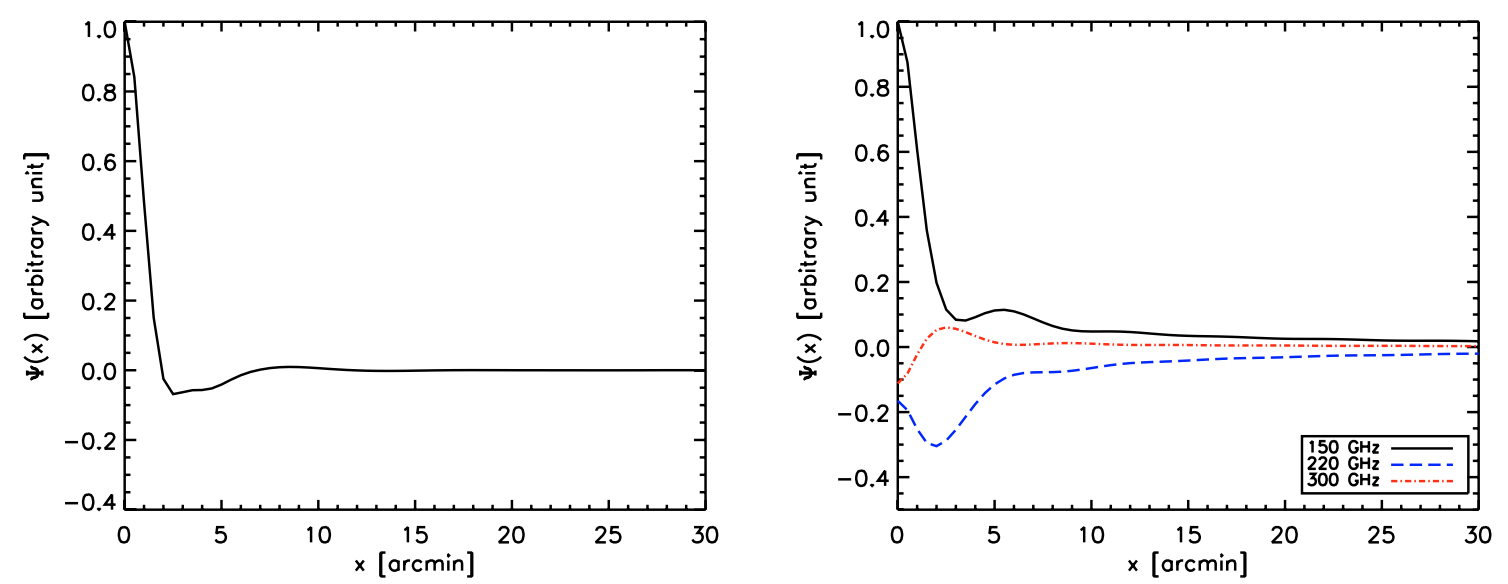

Fig. 1. Radial profiles of single frequency and multiple frequency matched filters for a cluster of $\theta=1$ arcmin. In the left-hand panel we see the spatial weighting used by the single frequency filter to optimally extract the cluster from the background (radio point sources + CMB) and noise. The filter is arbitrarily normalized to unity at the origin and the beam has a 2 arcmin FWHM. The right-hand panel shows the elements of the 3-band filter with 150, 220 and $300 \mathrm{GHz}$ channels. The three functions are arbitrarily normalized to the peak value of the $150 \mathrm{GHz}$ filter (black curve), and each channel has a 1 arcmin FWHM beam. In this case we see how the filter uses both spatial and spectral information to optimally extract the cluster.

beam and $P_{i j}^{\text {sky }}$ gives the foreground power (non-SZ signal) between channels $i$ and $j$. As written, we assume uncorrelated instrumental noise between observation frequencies.

Our aim is to quantify the effect of point source confusion on cluster detection using this filter. To this end, we ignore diffuse Galactic emission, which is small on cluster scales, and only include primary CMB temperature anisotropy and point source fluctuations in the sky power $P_{i j}^{\text {sky }}$. For our numerical results, we adopt a standard flat concordance $\mathrm{CMB}$ power spectrum $\left(\Omega_{\Lambda}=1-\Omega_{\mathrm{M}}=0.7, h=0.7\right.$, e.g., Freedman et al. 2001; Spergel et al. 2003) and employ a cluster template based on the spherically symmetric $\beta$-model with core radius $\theta$ and $\beta=2 / 3$ : $T_{\theta}(\boldsymbol{x})=\left(1+|\boldsymbol{x}|^{2} / \theta^{2}\right)^{-(3 \beta-1) / 2}$.

Two examples of the matched filter for $\theta=1$ arcmin are given in Fig. 1, one for a single frequency survey with a 2 arcmin beam (left-hand panel) and the other for a 3-band filter with 1 arcmin beams at 150,220 and $300 \mathrm{GHz}$ (right-hand panel). The filters are circularly symmetric because we have chosen a spherical cluster model, and the figure shows their radial profiles. We clearly see the spatial weighting used by the single frequency filter to optimally extract the cluster from the noise and from the point source and CMB backgrounds. The multiple frequency filter $\boldsymbol{\Psi}_{\boldsymbol{\theta}}$ is a 3-element column vector containing filters for each individual frequency. Their radial profiles are shown in the right-hand panel arbitrarily normalized to the peak of the $150 \mathrm{GHz}$ filter. The map from each band is filtered by the corresponding function and the results are then summed to produce the final filter output. We see here how the filter uses both spectral and spatial weighting to optimally extract the cluster signal.

\section{Point sources}

Two different extragalactic point source populations affect $\mathrm{SZ}$ observations (see Fig. 2). At frequencies below $\sim 100 \mathrm{GHz}$, radio galaxies and quasars dominate the source counts, while at higher frequencies dusty IR galaxies become more important.
The spectral dependence of source flux density in both populations is often modeled as a power law, $S_{v} \propto v^{\alpha}$, with spectral index $\alpha$. Radio sources tend to have falling spectra with $\alpha<0$ (Herbig \& Readhead 1992; Taylor et al. 2001; Mason et al. 2003), but flat and inverted spectra with $\alpha \geq 0$ appear more prominent with observing frequency (Bennett et al. 2003; Trushkin 2003). At millimeter wavelengths we observe the dust emission of IR galaxies in the Rayleigh-Jeans with rising spectra characterized by $\alpha \sim 3-4$ (Vlahakis et al. 2004). As will be seen, we need information on these extragalactic sources down to mJy flux densities and below; unfortunately, neither the distribution of $\alpha$ nor the source counts are well known for either population at these flux densities and at frequencies of interest for SZ observations ( $10-300 \mathrm{GHz})$.

\subsection{Radio galaxies}

Bennett et al. (2003) summarize the radio source counts at $30-40 \mathrm{GHz}$ from WMAP $\left(S_{v}>1 \mathrm{Jy}\right)$, DASI $\left(S_{v}>100 \mathrm{mJy}\right.$, Kovac et al. 2002), VSA $\left(S_{v}>50 \mathrm{mJy}\right.$, Taylor et al. 2003; Cleary et al. 2005) and CBI ( $S_{v}>10 \mathrm{mJy}$, Mason et al. 2003). Fitting to these data, Knox et al. (2004) find

$\left.\frac{\mathrm{d} N}{\mathrm{~d} S_{v}}\right|_{\mathrm{r}}=\frac{N_{\mathrm{o}}}{S_{\mathrm{o}}}\left(\frac{S_{v}}{S_{\mathrm{o}}}\right)^{\gamma}$

with $N_{\mathrm{o}}=30 \mathrm{deg}^{-2}, S_{\mathrm{o}}=1 \mathrm{mJy}$ and $\gamma=-2.0$. The integrated counts obtained from Eq. (6) are shown in Fig. 2 as the lower solid red line, together with the observations from $\mathrm{CBI}$ and the VSA. The model fits the CBI counts well, but lies high relative to the VSA counts at the bright end; as shown in Bennett et al. (2003), the counts in fact steepen toward higher flux densities, so a pure power law only matches the data over a limited range.

We will be interested in the counts at flux densities near $100 \mu \mathrm{Jy}$ for calculating the expected confusion noise in upcoming SZ surveys. This requires an extrapolation of the observed counts using Eq. (6) to much lower flux densities, which we 


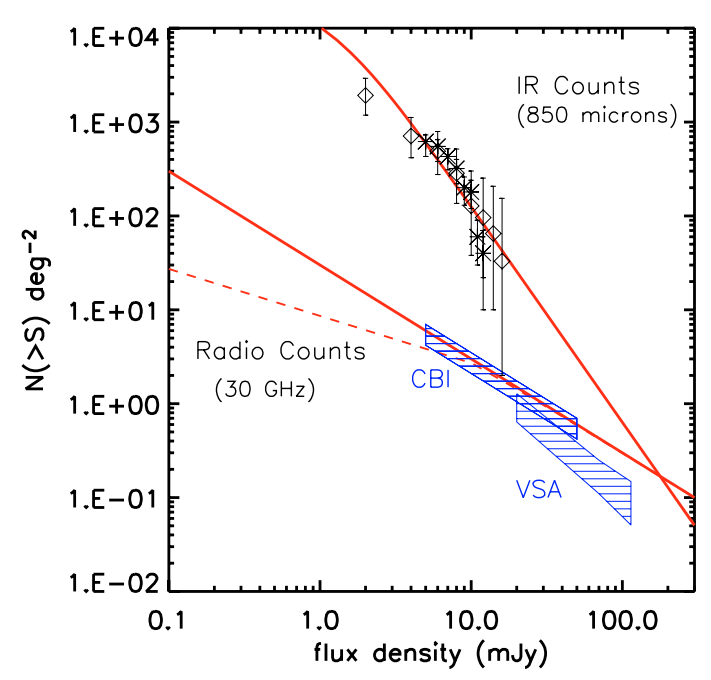

Fig. 2. Integrated radio and IR source counts. The lower solid red line results from the differential counts of Eq. (6), while the dashed red line corresponds to the model of Eq. (7). Measured counts at $30 \mathrm{GHz}$ from CBI and the VSA are shown as the hashed blue boxes. The upper solid red line gives the submillimeter source counts from Eq. (8). The diamonds show the measured counts at $850 \mu \mathrm{m}$ from the HDF-North (Borys et al. 2003) and the stars those from the $8 \mathrm{mJy}-$ survey (Scott et al. 2002).

view with caution. To get a handle on the uncertainty associated with this extrapolation, we consider an alternate model with a flattening slope toward the faint end:

$\left.\frac{\mathrm{d} N}{\mathrm{~d} S_{v}}\right|_{r^{\prime}}=\frac{N_{\mathrm{o}}}{S_{\mathrm{o}}}\left(1+\frac{S_{v}}{S_{\mathrm{o}}}\right)^{\gamma}$

with the same values of $N_{\mathrm{o}}, S_{\mathrm{o}}$ and $\gamma$. These counts are shown as the red dashed line in Fig. 2, which clearly provides an equally satisfactory fit to the observations. Comparing the confusion noise in the two models will give us a sense of the uncertainty in our estimates.

We adopt Eq. (6), alternatively Eq. (7), for the counts at $v=$ $30 \mathrm{GHz}$. Extrapolation to other frequencies suffers from uncertainty in the spectral index $\alpha$ of the emission law. Typically negative, determinations of $\alpha$ spread over a wide range, including positive values. Mason et al. (2003), for example, find an average $\langle\alpha\rangle=-0.45$, between 1.4 and $30 \mathrm{GHz}$, with a dispersion $\sigma_{\alpha}=0.37$, which is roughly consistent with the observations of Taylor et al. (2001) between 1.4 and $15 \mathrm{GHz}$. The brighter sources seen over the higher frequency WMAP bands, on the other hand, show much flatter spectra, with a distribution centered on $\alpha=0$ and a dispersion of $\sigma_{\alpha} \sim 0.3$ (Bennett et al. 2003; see also Trushkin 2003). There is a pressing need for better understanding of the radio source population at CMB frequencies.

\subsection{IR galaxies}

Dusty infrared luminous galaxies dominate the source counts at frequencies near $100 \mathrm{GHz}$ and higher. The dust in these galaxies is typically heated to temperatures of several tens of Kelvin by their interstellar radiation field. Its emission can be characterized with a blackbody spectrum modified by a power-law emissivity: $S_{v} \propto v^{\beta} B_{v}(T)$, where $\beta \sim 1-2$. In the Rayleigh-Jeans this leads to a steeply rising power-law with spectral index $\alpha \sim 3-4$, from which we see that source confusion from the IR population rises rapidly with frequency, the implications of which were recently discussed by White \& Majumdar (2004).

Blank field counts around $10 \mathrm{mJy}$ at $850 \mu \mathrm{m}$ where obtained by Scott et al. (2002) and Borys et al. (2003) using the SCUBA instrument. As discussed by the latter authors, the counts are well described by a double power-law:

$\left.\frac{\mathrm{d} N}{\mathrm{~d} S_{v}}\right|_{\mathrm{ir}}=\frac{N_{\mathrm{o}}}{S_{\mathrm{o}}}\left[\left(\frac{S_{v}}{S_{\mathrm{o}}}\right)^{1.0}+\left(\frac{S_{v}}{S_{\mathrm{o}}}\right)^{3.3}\right]^{-1}$

with $N_{\mathrm{o}}=1.5 \times 10^{4} \mathrm{deg}^{-2}$ and $S_{\mathrm{o}}=1.8 \mathrm{mJy}$. This model is shown in Fig. 2 as the upper solid red line, along with data taken from the two surveys.

The SCUBA Local Universe Galaxy Survey (SLUGS, Vlahakis et al. 2004) finds a broad distribution for the dust emissivity index with $\langle\beta\rangle \sim 1$ and a dispersion we take to be $\sigma_{\beta} \sim 0.2$. According to the SLUGS observations, optical galaxies tend to have lower spectral indexes than IRAS-selected objects; we eye-balled the above numbers to be representative of the population as a whole.

\section{Point source confusion}

Point source confusion is caused by random fluctuations in the number of unresolved sources in the filter. We now study the contribution of this confusion to the overall filter noise, $\sigma_{\theta}$, as a function of filter scale, $\theta$. As mentioned above, we only consider uncorrelated instrumental noise, primary CMB anisotropy and point source terms to the power spectrum matrix $\boldsymbol{P}$, whose off-diagonal elements are then just sky terms (to be multiplied by the beam):

$P_{i j}^{\mathrm{sky}}(k)=C_{l=|k|}+P_{i j}^{\mathrm{ps}}$

where $C_{l}$ is the CMB temperature angular power spectrum and $P_{i j}^{\mathrm{ps}}$ is the point source term. We quote power in units of CMB temperature equivalent and ignore spatial correlations of the point sources, which means that $P_{i j}^{\mathrm{ps}}$ is independent of $k$.

To calculate the point source terms $P_{i j}^{\mathrm{ps}}$, we adopt the counts of Eq. (6), alternatively Eq. (7), at $v_{\mathrm{r}} \equiv 30 \mathrm{GHz}$ and those of Eq. (8) at $v_{\text {ir }} \equiv 350 \mathrm{GHz}(850 \mu \mathrm{m})$. Unless otherwise specified, spectral indexes follow Gaussian distributions with $(\langle\alpha\rangle=0$, $\left.\sigma_{\alpha}=0.3\right)$ for radio sources, and $\left(\langle\alpha\rangle=3, \sigma_{\alpha}=0.2\right)$ for IR galaxies (see previous section). Then we have

$P_{i j}^{\mathrm{ps}}=\sigma_{\mathrm{r}}^{2} \cdot R_{i j}^{\mathrm{r}} G_{\mathrm{r}}\left(\sqrt{v_{i} v_{j}} / v_{\mathrm{r}}\right)+\sigma_{\mathrm{ir}}^{2} \cdot R_{i j}^{\mathrm{ir}} G_{\mathrm{ir}}\left(\sqrt{v_{i} v_{j}} / v_{\mathrm{ir}}\right)$

where (Scheuer 1957; Condon 1974)

$$
\left.\sigma_{(\mathrm{r}, \mathrm{ir})}^{2} \equiv\left(\frac{\partial B_{\left(v_{\mathrm{r}}, v_{\mathrm{ir}}\right)}}{\partial T}\right)^{-2} \int \mathrm{d} S_{v} S_{v}^{2} \frac{\mathrm{d} N}{\mathrm{~d} S_{v}}\right|_{(\mathrm{r}, \mathrm{ir})}
$$

gives the sky temperature variance due to radio or IR sources at the fiducial frequencies $v_{\mathrm{r}}$ and $v_{\mathrm{ir}}$; conversion to $\mathrm{CMB}$ 
temperature units is made with the Planck function $B_{v}$ at $T_{\mathrm{cmb}}=$ $2.725 \mathrm{~K}$ (Mather et al. 1999). This also appears in the $R$-factors:

$R_{i j}^{(\mathrm{r}, \mathrm{ir})} \equiv \frac{\left(\partial B_{v_{i}} / \partial T\right)^{-1}\left(\partial B_{v_{j}} / \partial T\right)^{-1}}{\left(\partial B_{v_{(\mathrm{r}, \mathrm{r})}} / \partial T\right)^{-2}}$.

The function $G$ accounts for spectral variations:

$G_{(\mathrm{r}, \mathrm{ir})}(x) \equiv \int \mathrm{d} \alpha N_{(\mathrm{r}, \mathrm{ir})}(\alpha) x^{2 \alpha}$

with $N_{(\mathrm{r}, \mathrm{ir})}$ being the normal distribution for radio and IR spectral indexes, respectively. These results assume that there is no correlation between flux density $S_{v}$ and spectral index, $\alpha$, so that the joint distribution is separable.

\subsection{Single frequency surveys}

Single frequency surveys can only use spatial information to control point source confusion. Operating at $v=15 \mathrm{GHz}$ and $30 \mathrm{GHz}$, respectively, AMI and SZA will contend primarily with the radio source population; AMiBA, on the other hand, must deal with both radio and IR sources at $v=90 \mathrm{GHz}$. The former two interferometers include long baselines dedicated to identifying and removing point sources at high spatial frequency on the sky, where they are cleanly separated from the more extended cluster SZ emission. Point source confusion is then caused by the residual population below the subtraction threshold, $S_{\text {lim }}$.

Apart from the beam convolution, point source confusion contributes to the filter variance $\sigma_{\theta}^{2}$ in the same manner as instrumental noise. Using the counts of Eq. (6) we find for the confusion power:

$$
\begin{aligned}
P_{30,30}^{\mathrm{ps}} & =\sigma_{\mathrm{r}}^{2}=(12 \mu \mathrm{K} \text { arcmin })^{2}\left(\frac{S_{\text {lim }}}{100 \mu \mathrm{Jy}}\right) \\
P_{15,15}^{\mathrm{ps}} & =\sigma_{\mathrm{r}}^{2} \cdot R_{15,15}^{\mathrm{r}} \cdot G_{\mathrm{r}}(1 / 2) \\
& >(48 \mu \mathrm{K} \text { arcmin })^{2}\left(\frac{S_{\text {lim }}}{100 \mu \mathrm{Jy}}\right)
\end{aligned}
$$

where the numerical value in the last line assumes $G_{\mathrm{r}}(1 / 2)=1$, while it most certainly is larger. These values are comparable to target sensitivities for interferometer surveys, implying that these instruments will have to subtract sources down to $\sim 100 \mu \mathrm{Jy}$ or better. Assuming a $\theta_{\mathrm{fwhm}} \sim 2$ arcmin synthesized beam, we calculate the confusion limit on the integrated Compton $Y$ parameter for unresolved clusters. We conclude that confusion from sources below $100 \mu \mathrm{Jy}$ will limit SZ sensitivity to $Y \sim 3 \times 10^{-6} \operatorname{arcmin}^{2}$ at $30 \mathrm{GHz}$ and $Y \sim 10^{-5} \operatorname{arcmin}^{2}$ at $15 \mathrm{GHz}$ (both are at $1 \sigma$ ). The former limit is a factor $\sim 5$ greater than the confusion expected from primary CMB anisotropy in the concordence model for unresolved clusters (Melin et al. 2005).

Adopting the alternative model of Eq. (7), these confusion limits drop by a factor $\sim 20$, and the dependence of the variance on the source subtraction threshold approaches $\propto S_{\text {lim }}^{3}$. There is clearly a large uncertainty associated with extrapolation of the counts to faint flux densities. In this light, note that as long as the counts do not steepen toward lower flux densities, the confusion estimates are, fortunately, dominated by the counts at $S_{\text {lim }}$, rather than at some unknown cut-off at even fainter levels.

\subsection{Multiple frequency surveys}

Planned bolometer-based surveys will operate over several millimeter and submillimeter bands, allowing them to use spectral information to extract clusters from the foregrounds. This will in fact be their only way to reduce the effects of point source confusion, because they will not have the spatial resolution ${ }^{4}$ needed for subtracting point sources from cluster images. In the multi-frequency case, we refer to the optimal spatio-spectral filter as a multi-filter.

Specifically, the multi-filter performs a weighted sum designed to remove foregrounds from the SZ cluster signal, as illustrated in the right-hand panel of Fig. 1. Figure 3 helps to understand the filter's workings and it will allow us to draw some important conclusions. The figure shows the filter noise $\sigma_{\mathrm{Y}}(1 \sigma)$ in terms of the integrated Compton $Y$ parameter as a function of filter scale for various band and foreground combinations. This is calculated from the filter variance as $\sigma_{\mathrm{Y}}=\sigma_{\theta} \int \mathrm{d}^{2} x T_{\theta}(\boldsymbol{x})$. We take as representative of planned observations a survey with $10 \mu \mathrm{K}$ instrumental noise per 1 arcmin lobe (FWHM) in all bands. We only include the IR source population (dominant at these frequencies) below $S_{\text {lim }}=100 \mathrm{mJy}$, assuming brighter sources are explicitly subtracted; this is well above the knee in the differential counts of Eq. (8). We further fix, for the present, the spectral index to $\alpha=3$ with zero dispersion $(G=1)$.

Consider first the case with just instrumental noise, point sources (no CMB) and two frequencies, one at $v=150 \mathrm{GHz}$ and the other at the thermal SZ null, $v=220 \mathrm{GHz}$. Point source confusion is severe in each individual band, as illustrated by Fig. 4. The dotted line in the left panel of Fig. 3 shows the total filter noise with just the $150 \mathrm{GHz}$ band. When both bands are used in the filter, the filter noise $\sigma_{\mathrm{Y}}$ drops considerably (red dot-dashed line), approaching the pure instrumental noise limit for the 2-band filter, shown as the black dashed-3 dotted line. It is straightforward to show under these circumstances that the filter performs a direct subtraction of the point source signal from the $150 \mathrm{GHz}$ channel by extrapolation of the $220 \mathrm{GHz}$ signal using the known spectral index $\alpha$. What we see here is that the subtraction is perfect to the instrumental noise limit.

The sky of course also includes the CMB signal and other foregrounds, and the point source spectral index has a non-zero distribution, both of which complicate the subtraction. We discuss the second effect below and now add CMB anisotropy, keeping $\alpha$ fixed. The 2-band filter is no longer able to separately determine the three sky signals (SZ, point sources and CMB), with as a consequence a significant rise in total SZ noise, as shown by the solid green line. Surprisingly, the situation does not improve even when we include more information with a third observing band at $v=300 \mathrm{GHz}$ (blue dashed line).

This interesting result is due to the fact that both the 220 and $300 \mathrm{GHz}$ bands are dominated by point source

\footnotetext{
${ }^{4}$ Most planned surveys have arcminute resolution.
} 



Fig. 3. Noise $(1 \sigma)$ on the integrated Compton $Y$ parameter as a function of filter scale. We assume $10 \mu \mathrm{K}$ noise/beam and adopt 1 arcmin beams in all bands, except for the green 3-dot-dashed curve in the right-hand panel. At these frequencies, we only include IR sources below $S_{\lim }=100 \mathrm{mJy}$, following the counts of Eq. (8), and with a spectral index $\alpha=3$ and no dispersion. Left panel: the black dotted line shows the sensitivity at $150 \mathrm{GHz}$, where the instrumental noise and point source confusion are comparable (see Fig. 4). Confusion is effectively eliminated by a 2-band filter, as demonstrated by the red dot-dashed line lying just above the pure instrumental noise limit (black 3 dot-dashed curve). The upper solid green line shows the result when adding primary CMB anisotropy, in which case the 2-band filter is unable to cleanly separate the three astrophysical components. Addition of a third band at $300 \mathrm{GHz}$ (blue dashed line) does not greatly improve the result, because both the 220 and $300 \mathrm{GHz}$ channels are dominated by point sources, as shown in Fig. 4 (see text). Right panel: artificially removing the point sources from the $300 \mathrm{GHz}$ channel, we see (black dotted line) that the filter gains enough leverage on the three astrophysical signals to drop its noise to the instrumental limit (red dot-dashed line). Source confusion being greatly reduced at lower frequencies, a 3-band filter with $90 \mathrm{GHz}$ (solid green line) performs better; this remains true even when we degrade the $90 \mathrm{GHz}$ beam to a more realistic 2 arcmin FWHM (green 3-dot-dashed curve).

confusion (see Fig. 4) - neither provides good information the $\mathrm{CMB}$ anisotropy, so the filter remains unable to completely separate the three sky signals. We can test this conclusion by artificially removing point sources from the new channel at $300 \mathrm{GHz}$. The result is shown in the right-hand panel of the figure as the black dotted line; the total SZ noise $\sigma_{\mathrm{Y}}$ drops to a level comparable to the level induced just by instrumental noise (red dot-dashed line), indicating that once again the subtraction is almost perfect.

This has important consequences for SZ surveying. Observing at high frequencies, such as $300 \mathrm{GHz}$, is very difficult from the ground due to atmospheric effects; moving up in frequency, one approaches strong water vapor lines. What we have just seen suggests that including bands beyond the thermal SZ null may not be worth the effort, at least not for detecting clusters.

To further explore this issue, we replace the $300 \mathrm{GHz}$ channel by a $90 \mathrm{GHz}$ band. At this lower frequency, point source confusion is greatly reduced and gives the filter a better handle on CMB anisotropy; in fact, as shown in Fig. 4, point source confusion is well below the instrumental noise level at $90 \mathrm{GHz}$. The green solid line in the right-hand panel of Fig. 3 shows the new result: this three-band filter with $90 \mathrm{GHz}$ performs significantly better than the one with $300 \mathrm{GHz}$. It is, however, unlikely that all three bands will have the same beam size, which we have taken as 1 arcmin throughout this discussion.
The green 3-dot-dashed curve in the right-hand panel of the figure shows the result for a three-band filter with a 2 arcmin beam at $90 \mathrm{GHz}$. Even with the larger beam at the lower frequency, the result remains qualitatively the same - the filter with $90 \mathrm{GHz}$ performs better than the one with $300 \mathrm{GHz}$.

Multi-frequency surveys will certainly include 150 and $220 \mathrm{GHz}$ bands to cover the maximum decrement and null of the thermal SZ signal. We conclude here that a $90 \mathrm{GHz}$ band is a more valuable addition than one at $300 \mathrm{GHz}$ for cluster extraction, despite a loss in angular resolution at the lower frequency.

As a final note, we consider the effects of dispersion in the source spectral index $\alpha$. With dispersion, the filter can no longer perform a perfect subtraction by extrapolation across bands; it must instead find appropriate frequency weights for a statistically optimal subtraction. In the point source power spectrum matrix $P_{i j}^{\mathrm{ps}}$, only the self power $P_{v_{\mathrm{ir}}, v_{\mathrm{ir}}}^{\mathrm{ps}}$ remains unaffected; other diagonal elements will increase, while correlations between bands (in the off-diagonal elements) decrease. We therefore expect the filter's performance to decline.

We examined the importance of this effect using the function $G_{\text {ir }}$ defined in Eq. (13). Dispersions up to $\sigma_{\alpha}=0.5$ increase matrix elements involving the 150 and $220 \mathrm{GHz}$ bands of the power spectrum matrix by at most $20 \%$, relative to their values with zero dispersion. The $90 \mathrm{GHz}$ channel is of course the most affected: the auto-power element $P_{90,90}^{\mathrm{ps}}$ increases by more 




Fig. 4. Power spectra of the primary CMB anisotropy (solid black line), point source confusion in different bands (as labeled) and instrumental noise; the latter corresponds to $10 \mu \mathrm{K}$ noise $/ 1$ arcmin lobe, which we take here to be the same in all bands. Only IR sources with $S_{v}<S_{\lim }=100 \mathrm{mJy}$ are included, according to the counts of Eq. (8), and with $\alpha=3$ and no dispersion. The figure shows the that the $300 \mathrm{GHz}$ channel is dominated by sources, explaining why the 3-band filter with $90 \mathrm{GHz}$ performs more efficiently than the one with $300 \mathrm{GHz}$ (see Fig. 3).

than $50 \%$ for the same dispersion. Nevertheless, when running the filter combinations of Fig. 3, we find only a small change in the filter noise curves, barely perceptible by eye. We conclude that even rather large variations in the frequency dependence of individual source spectra does not significantly increase confusion noise through the filter. In more general terms, this also suggests that our confusion estimates are not strongly dependent on the foreground model.

\section{Discussion and conclusion}

Primary CMB anisotropy and extragalactic point sources are the most important foregrounds for SZ surveys. Point source confusion is a particularly critical issue because it rises rapidly on cluster angular scales. We have quantified its importance for both single frequency and multiple frequency surveys using current estimates of the radio and IR source counts and an optimal matched multi-filter for cluster extraction. Our main conclusion are:

- The expected confusion level from radio point sources is uncertain due to lack of information on the counts at required flux densities: a power-law extrapolation of the observed counts (Eq. (6)) leads to a confusion-limited SZ sensitivity at $30 \mathrm{GHz}$ of $Y \sim 3 \times 10^{-6}$ arcmin $^{2}$ from unresolved sources below $S_{\lim }=100 \mu \mathrm{Jy}$ ( $1 \sigma$ for clusters unresolved by a 2 arcmin beam); at $15 \mathrm{GHz}$ the corresponding sensitivity is $Y \sim 10^{-5} \operatorname{arcmin}^{2}$. An alternative model in which the counts flatten at low flux density (Eq. (7)) predicts much lower confusion limits, reduced by a factor

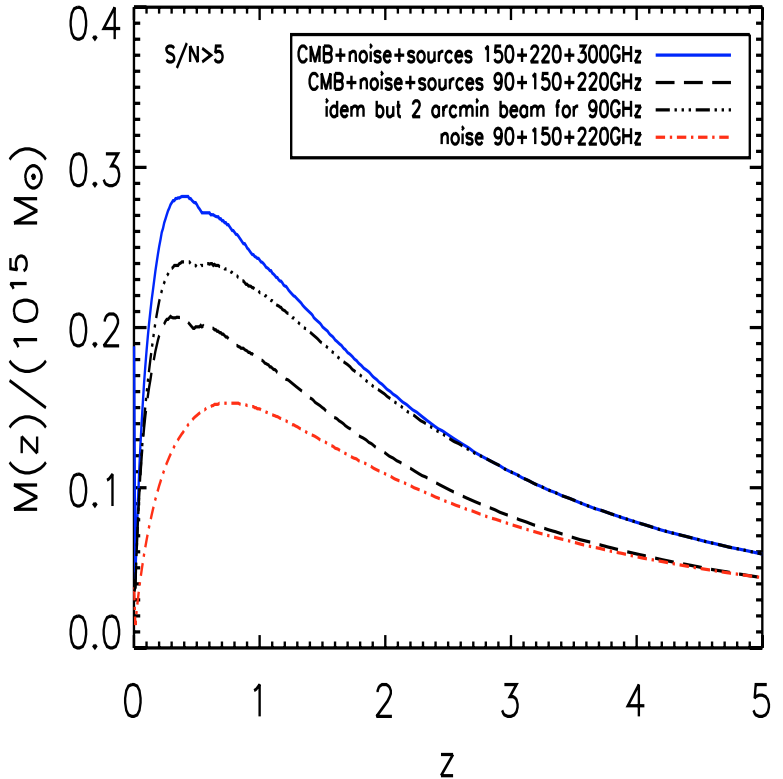

Fig. 5. Minimum detectable mass as a function of redshift. The upper solid blue curve shows the result for the 3-band filter with a $300 \mathrm{GHz}$ channel, while the middle dashed black curve gives the result when this channel is replaced by a $90 \mathrm{GHz}$ band. For reference, the lower red dash-dotted curve gives the ideal detector-noise limited detection mass for the 3-band filter with $90 \mathrm{GHz}$. The 3-band filter with $90 \mathrm{GHz}$ shows its higher sensitivity (see Fig. 3), but still suffers from residual foreground (CMB) contamination. These results follow for 1 arcmin FWHM beams in all bands. For comparison, the black 3-dot-dashed line gives the mass limit for the 3-band filter with a $90 \mathrm{GHz}$ beam of 2 arcmin FWHM, which continues to perform better than the 3-band filter with $300 \mathrm{GHz}$ despite the loss of angular resolution at the lower frequency.

of 20 , in which case CMB confusion becomes the limiting factor (Melin et al. 2005).

- Currently planned multiple frequency bolometer surveys (e.g., $10 \mu \mathrm{K}$ instrumental noise/ 1 arcmin beam) greatly reduce the large confusion noise from IR sources and can attain $1 \sigma$ sensitivity of $Y \sim 7 \times 10^{-6} \operatorname{arcmin}^{2}$ for unresolved clusters (see Fig. 3). Nevertheless, both residual point source and $\mathrm{CMB}$ anisotropy confusion significantly increase the total filter noise, degrading survey sensitivity from the ultimate instrumental noise limit.

- For multiple frequency surveys, observation bands below the thermal SZ null (e.g., 90+150+220 GHz) are more efficient for cluster extraction than observations at higher frequencies (e.g., 150+220+300 GHz), despite a potential loss of angular resolution in the $90 \mathrm{GHz}$ channel (e.g., 2 arcmin beam instead of our fiducial 1 arcmin beam).

The minimum detectable cluster mass as a function of redshift, $M_{\text {det }}(z)$, is a key characteristic of a survey. To further illustrate the last point, we show $M_{\text {det }}(z)$ in Fig. 5 for the 3-band multifilter. In making this figure, we adopted simple self-similar relations for $Y(M, z)$ and $\theta_{\mathrm{c}}(M, z)$ (Melin et al. 2005); given the potentially important theoretical uncertainty in these relations, the absolute positioning of the curves should be viewed with caution - more robust and more pertinent to our discussion are 
their relative positions. The 3-band filter with $90 \mathrm{GHz}$ (black dashed line) gains mass sensitivity compared to the filter with $300 \mathrm{GHz}$ (solid blue line), assuming 1 arcmin beams in all bands. It does not, however, reach the ideal noise limit (red dot-dashed line), due to residual point source and CMB confusion through the filter. A 3-band filter with lower angular resolution at $90 \mathrm{GHz}$ ( 2 arcmin beam), a more probable observing situation, does somewhat worse, but still better than the 3-band filter with $300 \mathrm{GHz}$ (and 1 arcmin beams in all bands). This is an important consideration given the difficulty imposed by atmospheric effects on observations at high frequencies.

With the $S / N>5$ detection threshold [i.e., $5 \sigma_{\mathrm{Y}}(\theta)$ ], we find 15 clusters $/ \mathrm{deg}^{2}$ for the filter with $300 \mathrm{GHz}(1$ arcmin all bands), 30 clusters $/ \mathrm{deg}^{2}$ for the filter with $90 \mathrm{GHz}$ and 1 arcmin beams, and 18 clusters/ $/ \mathrm{deg}^{2}$ for the 3-band filter with a 2 arcmin beam at $90 \mathrm{GHz}$. There are 47 detected clusters/ $/ \mathrm{deg}^{2}$ at the ultimate noise-limit of the 3-band filter with a 1 arcmin beam at $90 \mathrm{GHz}$. These numbers should be compared to the 85 clusters $/ \mathrm{deg}^{2}$ with mass $>10^{14}$ solar masses with our model parameters.

An important consequence of our results is that SZ survey selection functions are affected by residual astrophysical confusion and are not uniquely determined by instrumental properties. Specifically, we have seen that even a 3-band bolometer survey with good angular resolution and optimal filter cluster extraction experiences a mixture of residual point source and primary CMB confusion. Cluster catalog construction will therefore suffer from uncertaintly in astrophysical foreground modeling (Melin et al. 2005).

In conclusion, our results support the expectation that future ground-based SZ surveys will provide rich cluster cata$\operatorname{logs}$ for cosmological studies. Confusion from point sources and primary CMB anisotropy can be greatly reduced by multifrequency bolometer surveys, but some residual point source and CMB anistropy confusion noise will affect cluster detection and catalog construction.

Acknowledgements. We thank G. Evrard and the organizers of the Future of Cosmology with Clusters of Galaxies conference, where this work began in earnest. Thanks to J. Mohr for encouragement and to the anonymous referee who helped improve the clarity of the presentation. J.-B. Melin was supported at U.C. Davis by the National Science Foundation under Grant No. 0307961 and NASA under Grant No. NAG5-11098.

\section{References}

Aghanim, N., Hansen, S. H., \& Lagache, G. 2005, A\&A, 439, 901

Barbosa, D., Bartlett, J. G., \& Blanchard, A. 1996, A\&A, 314, 13

Bartlett, J. G. 2001, review in Tracing cosmic evolution with galaxy clusters (Sesto Pusteria 3-6 July 2001), ASP Conf. Ser., in press [arXiv: astro-ph/0111211]

Bennett, C., Hill, R. S., Hinshaw, G., et al. 2003, ApJS, 148, 97

Benson, A. J., Reichardt, C., \& Kamionkowski, M. 2002, MNRAS, 331,71

Birkinshaw, M. 1999, Proc. 3K Cosmology, 476, American Institute of Physics, Woodbury, 298
Borys, C., Chapman, S., Halpern, M., \& Scott, D. 2003, MNRAS, 344, 385

Carlstrom, J. E., Holder, G. P., \& Reese, E. D. 2002, ARA\&A, 40, 643

Cleary, K. A., Taylor, A. C., Waldram, E., et al. 2005, MNRAS, 360, 340

Colafrancesco, S., Mazzotta, P., \& Vittorio, N. 1997, ApJ, 488, 566

Condon, J. J. 1974, ApJ, 188, 279

Eke, V. R., Cole, S., \& Frenk, C. S. 1996, MNRAS, 282, 263

Freedman, W. L., Madore, B. F., Gibson, B. K., et al. 2001, ApJ, 553, 47

Haehnelt, M. G., \& Tegmark, M. 1996, MNRAS, 279, 545

Haiman, Z., Mohr, J. J., \& Holder, G. 2001, ApJ, 553, 545

Herbig, T., \& Readhead, A. C. S. 1992, ApJS, 81, 83

Herranz, D., Sanz, J. L., Hobson, M. P., et al. 2002, MNRAS, 336, 1057

Holder, G. P., Mohr, J. J., Carlstrom, J. E., Evrard, A. E., \& Leitch, E. M. 2000, ApJ, 544, 629

Kneissl, R., Jones, M. E., Saunders, R., et al. 2001, MNRAS, 328, 783

Knox, L., Holder, G., \& Church, S. 2004, ApJ, 612, 96

Kovac, J., Leitch, E. M., Pryke, C., et al. 2002, Nature, 420, 772

Mason, B. S., Pearson, T. J., Readhead, A. C. S., et al. 2003, ApJ, 591, 540

Mather, J. C., Fixsen, D. J., Shafer, R. A., et al. 1999, ApJ, 512, 511

Melin, J.-B., Bartlett, J. G., \& Delabrouille, J. 2005, A\&A, 429, 417

Melin, J.-B., et al. 2006, in preparation

Schäfer, B. M., Pfrommer, C., Hell, R. M., \& Bartelmann, M. 2004 [arXiv: astro-ph/0407090]

Scheuer, P. A. G. 1957, Proc. Camb. Phil. Soc., 53, 764

Scott, S. E., Fox, M. J., Dunlop, J. S., et al. 2002, MNRAS, 331, 817

Spergel, D. N., Verde, L., Peiris, H. V., et al. 2003, ApJS, 148, 175

Sunyaev, R. A., \& Zel'dovich, Ya. B. 1970, Comments Astrophys. Space Phys., 2, 66

Sunyaev, R. A., \& Zel'dovich, Ya. B. 1972, Comments Astrophys. Space Phys., 4, 173

Taylor, A. C., Grainge, K., Jones, M. E., et al. 2001, MNRAS, 327, L1

Taylor, A. C., Carreira, P., Cleary, K., et al. 2003, MNRAS, 341, 1066

Trushkin, A. S. 2003, Bull. Spec. Astrophys. Obs. N. Caucasus, 55, 90 [arXiv: astro-ph/0307205]

Vlahakis, C., Dunne, L., \& Eales, S. 2004 [arXiv: astro-ph/0411067]

Wang, S., Khoury, J., Haiman, Z., \& May, M. 2004, Phys. Rev. D, 70, 123008

Weller, J., \& Battye, R. A. 2003, NewAR, 47, 775

White, M., \& Majumdar, S. 2004, ApJ, 602, 565

Web pages of various SZ experiments:

- ACBAR http://cosmology.berkeley.edu/group/swlh/ acbar/

- ACT http://www.hep. upenn.edu/ angelica/act/act.html

- AMI http://www.mrao.cam.ac.uk/telescopes/ami/ index.html

- AMiBA http://www.asiaa.sinica.edu.tw/amiba

- APEX http://bolo.berkeley.edu/apexsz

- SPT http://astro.uchicago.edu/spt/

- SZA http://astro.uchicago.edu/sze

- Olimpo http://oberon.roma1.infn.it/

- Planck http://astro.estec.esa.nl/Planck/ 